Methods Using a United Kingdom general population database, we conducted a cohort study of adult SLE patients, identified by Read codes. The exposures of interest were opioid prescriptions between January 1, 2007 and December 31, 2016. We classified opioids as weak, including tramadol and codeine, and strong, including hydrocodone, morphine, fentanyl, oxycodone, hydromorphone, and methadone. We examined the proportion of patients receiving prescriptions for weak and strong opioids and performed logistic regression to assess whether prescription use of these medications varied by age, sex, duration of SLE, other medication use for SLE, lifestyle exposures (alcohol and tobacco use), and socioeconomic status. We adjusted for age and sex.

Results Of 10,784 SLE patients, (86\% female, mean age 51.2 years), 32\% were ever prescribed weak opioids (tramadol or codeine) and $10 \%$ were ever prescribed strong prescription opioids during the study period. $21 \%$ and $7 \%$ received multiple prescriptions for weak and strong opioids, respectively. Prescription opioid use was more common among older patients (adjusted OR 2.27 [95\% CI 1.91-2.70] for weak opioid use and 2.94 [95\% CI 2.14-4.03] for strong opioid use among patients over age 50 compared with those under age 30. (Table 1). SLE patients who were also taking NSAIDs, DMARDs, or glucocorticoids each had an increased odds of receiving prescription opioids. Current smokers were also more likely to be prescribed prescription opioids. There was no significant association found between deprivation score, a measure of socioeconomic status, and opioid usage.

Conclusions In this general population-based cohort study, nearly one-third of SLE patients were ever prescribed weak opioids and $10 \%$ were ever prescribed stronger opioids. This rate of opioid prescription use is higher among patients who are also taking NSAIDs, glucocorticoids, and DMARDs. These findings indicate the use of these potentially dangerous medications among a substantial portion of SLE patients. Future studies should assess the impact of opioid usage on mortality and other important outcomes among patients with SLE.

Funding Source(s): NIH/NIAMS T32-AR-007258

\section{CLINICAL AND SEROLOGICAL CORRELATIONS OF AUTOANTIBODIES DIRECTED AGAINST RNP-C IN SYSTEMIC LUPUS ERYTHEMATOSUS}

${ }^{1}$ May Choi ${ }^{*},{ }^{2}$ Ann E Clarke, ${ }^{3}$ Michelle Jung, ${ }^{2}$ Claire Barber, ${ }^{4}$ Yvan St Pierre, ${ }^{5}$ Michael Mahler, ${ }^{3}$ Marvin Fritzler. ${ }^{1}$ Cumming School of Medicine, University of Calgary; ${ }^{2}$ Division of Rheumatology, Cumming School of Medicine, University of Calgary; ${ }^{3}$ University of Calgary; ${ }^{4}$ Department of Medicine, Division of Rheumatology, Faculty of Medicine, McGill University; ${ }^{5}$ nova Diagnostics

\subsection{6/lupus-2019-Ism.158}

Background Autoantibodies to RNP-C protein, along with RNP$A$, is a component of the U1RNP macromolecular complex. Antibodies to U1-RNP are typically associated with mixed connective tissue disease and other systemic autoimmune rheumatic diseases. Autoantibodies to RNP-C or their clinical significance have not been thoroughly studied in systemic lupus erythematosus (SLE). The goals of this study were to determine the frequency of anti-RNP-C autoantibodies in a SLE cohort and identify demographic, clinical, and serologic correlations.

Methods Patients fulfilling the ACR or SLICC Classification Criteria for SLE were enrolled in a local cohort. Demographic, clinical information (disease activity SLEDAI-2K; damage SLICC/ACR Damage Index (SDI)), and sera were collected at time of enrollment. Antibodies to anti-RNP-C were determined by a line immunoassay using a purified, full-length recombinant protein Euroimmun GmbH, Luebeck, Germany). Univariable and multivariable analysis were performed to determine associations between the prevalence of high positive anti-RNP-C and demographic (age, sex, race/ethnicity), clinical features (SLICC/ACR classification criteria, SLEDAI-2K and SDI total scores and subscales from SLEDAI-2K), medications, and other autoantibodies.

Results 138 SLE patients were included; 89.1\% were female with a mean age of 46.1 years (SD 18.1 years) and disease duration of 13.7 years (SD 11.6 years). The prevalence of antiRNP-C antibodies was 19.6\% (27/138); 25.9\% (7/27) were male. Univariable analysis demonstrated that patients fulfilling a higher total SLICC criteria (Odds Ratio, OR, 1.4 [95\% Confidence interval, CI: 1.1-1.7]), particularly maculopapular rash (OR 4.0 [95\% CI: 1.3-11.9]) and pericardial effusion (OR 6.3 [95\% CI: 1.3-29.9]), or a higher SLEDAI score (OR 1.2 [95\% CI: 1.0-1.3]) were more likely to be anti-RNP-C positive. Also, patients with higher immunological SLICC subscales (OR 1.8 [95\% CI: 1.2-2.5]), anti-dsDNA (OR 7.6 [95\% CI: 2.6-21.9]), anti-Sm (OR 29.7 [95\% CI: 9.8-89.9]), anti-RNP (OR 44.2 [95\% CI: 12.0-163.4]), anti-nucleosome (OR 9.4 [95\% CI: 2.5-35.0]), anti-Ribosomal P (OR 7.4 [95\% CI: 2.5-22.5]), or anti-RNP-A (OR 153.8 [95\% CI: 35.8-660.5]) were associated with anti-RNP-C positivity. Patients who were female (OR 0.2 [95\% CI: 0.1-0.7]), had longer disease duration (OR 0.9 [95\% CI: $0.9-1.0]$ ), or were on steroids (OR 0.3 [95\% CI: $0.1-0.7]$ ) were less likely to be anti-RNP-C positive. Multivariable analysis demonstrated that patients who were anti-RNP-A positive (OR 78.5 [95\% CI: 6.5-941.2]) were more likely to be antiRNP-C positive while those who were female (OR 0.1 [95\% CI: 0.01-1.0]) were less likely to be anti-RNP-C positive.

Conclusions Anti-RNP-C antibodies were common (19.6\%) in our SLE cohort. In SLE, they were associated with anti-RNPA antibodies, a finding which is in keeping with the concept of inter-molecular epitope spreading. Most notably, anti-RNP$\mathrm{C}$ antibodies were more likely seen to be seen in males with SLE. A thorough study of male SLE patients is needed.

Funding Source(s): The Arthritis Society Chair in Rheumatic Diseases at the Cumming School of Medicine, University of Calgary

\section{PEDIATRIC ONSET LUPUS WITH STEVENS JOHNSON SYNDROME/TOXIC EPIDERMAL NECROLYSIS: AN UNUSUAL ASSOCIATION}

${ }^{1}$ Himanshi Chaudhary*, ${ }^{2}$ Dharmagat Bhattarai, ${ }^{3}$ Pandiarajan Vignesh, ${ }^{4}$ Amit Rawat, ${ }^{5}$ Deepti Suri, ${ }^{4}$ Sunil Dogra, ${ }^{5}$ Surjit Singh. 'Allergy Immunology Unit, Advanced Pediatrics Center, PGIMER Chandigarh; ${ }^{2}$ PGIMER; ${ }^{3}$ Dept. of Pediatrics, Allergy- Immunology Unit, Postgraduate Institute of Medical Education and Research; ${ }^{4} P G I M E R$ Chandigarh; ${ }^{5}$ Postgraduate Institute of Medical Education and Research, Chandigarh, India

\subsection{6/lupus-2019-Ism.159}

Background The co-existence of SJS/TEN in context of childhood onset lupus is distinctly unusual and only a handful of cases have been reported in the literature. As the presentation of both illnesses can be heterogenous, simultaneous occurrence of both diseases in the same patient can pose diagnostic difficulties for the treating pediatrician.

Methods we report an 11 year old girl who presented with an SJS-TEN rash and had other features consistent with SLE. Results An 11-year-old girl presented with history of alopecia, intermittent fever, a maculopapular rash progressing into patchy bullous and desquamating lesions, red scaly eyes and 2 